\title{
On the Achievable Diversity-Multiplexing Tradeoff in Interference Channels
}

\author{
Cemal Akçaba and Helmut Bölcskei \\ Communication Technology Laboratory \\ ETH Zurich, Switzerland \\ Email: \{cakcaba | boelcskei\}@ nari.ee.ethz.ch
}

\begin{abstract}
We analyze two-user single-antenna fading interference channels with perfect receive channel state information (CSI) and no transmit CSI. For the case of very strong interference, we prove that decoding interference while treating the intended signal as noise, subtracting the result out, and then decoding the desired signal, a process known as "stripping", achieves the diversitymultiplexing tradeoff (DMT) outer bound derived in Akuiyibo and Lévêque, Int. Zurich Seminar on Commun., 2008. The proof is constructive in the sense that it provides corresponding code design criteria for DMT optimality. For general interference levels, we compute the DMT of a fixed-power-split Han and Kobayashi type superposition coding scheme, provide design criteria for the corresponding superposition codes, and find that this scheme is DMToptimal for certain multiplexing rates.
\end{abstract}

\section{INTRODUCTION}

The interference channel (IC) models the situation where $M$ unrelated transmitters communicate their separate messages to $M$ independent receivers, each of which is assigned to a single transmitter. Apart from a few special cases [1], [2], [3], the capacity region of the IC remains unknown. Recently, Etkin et al. [4], [5] showed that in the interference-limited regime, the capacity region of the IC is achievable to within one bit; later Telatar and Tse [6] generalized this result to a wider class of ICs. Shang et al. derived the noisy-interference sum-rate capacity for Gaussian ICs in [7], while Raja et al. [8] characterized the capacity region of the two-user finite-state compound Gaussian IC to within one bit. Annapureddy and Veeravalli [9] showed that the sum capacity of the two-user Gaussian IC under weak interference is achieved by treating interference as noise.

In [10], Akuiyibo and Lévêque derived an outer bound on the diversity-multiplexing tradeoff (DMT) region of fading ICs based on the results of Etkin et al. [5]. In this paper, we investigate the achievability of this outer bound and we analyze the DMT realized by a stripping decoder and a fixed-power-split Han and Kobayashi (HK)-type superposition coding scheme. For the sake of simplicity, throughout the paper, we restrict our attention to the two-user case. Furthermore, we assume that the receivers have perfect channel state information (CSI) whereas the transmitters only know the channel statistics. We would like to point out that the schemes used in [5] make explicit use of transmit CSI and so does the scheme in [10], which immediately implies that the results reported in [10] serve as an outer bound on the DMT achievable in the absence of transmit CSI, the case considered here. The contributions in this paper can be summarized as follows:
- For very strong interference in the sense of [5], we show that a stripping decoder which decodes interference while treating the intended signal as noise, subtracts the result out, and then decodes the intended signal is DMT-optimal. We furthermore find that the optimal-DMT can be achieved if each of the two users employs a code that is DMT-optimal on a single-input single-output (SISO) channel.

- For general interference levels, we compute the DMT of a two-message, fixed-power-split HK-type superposition coding scheme and provide design criteria for the corresponding superposition codes. We find that this scheme is DMT-optimal for certain multiplexing rates.

Notation: The superscripts ${ }^{T}$ and ${ }^{H}$ stand for transpose and conjugate transpose, respectively. $x_{i}$ represents the $i$ th element of the column vector $\mathbf{x}$, and $\lambda_{\min }(\mathbf{X})$ denotes the smallest eigenvalue of the matrix $\mathbf{X} . \mathbf{I}_{N}$ is the $N \times N$ identity matrix, and $\mathbf{0}$ denotes the all zeros matrix of appropriate size. All logarithms are to the base 2 and $(a)^{+}=\max (a, 0) . X \sim \mathcal{C N}\left(0, \sigma^{2}\right)$ stands for a circularly symmetric complex Gaussian random variable (RV) with variance $\sigma^{2} . f(\rho) \doteq g(\rho)$ denotes exponential equality of the functions $f(\cdot)$ and $g(\cdot)$, i.e., $\lim _{\rho \rightarrow \infty} \log [f(\rho)] / \log \rho=$ $\lim _{\rho \rightarrow \infty} \log [g(\rho)] / \log \rho$. The symbols $\dot{\geq}, \dot{\leq}, \dot{>}$ and $\dot{<}$ are defined analogously.

System model: We consider a two-user fading IC where two transmitters communicate information to two receivers via a common channel. The fading coefficient between transmitter $i(i=1,2)$ and receiver $j(j=1,2)$ is denoted by $h_{i j}$ and is assumed to be $\mathcal{C N}(0,1)$. Transmitter $i\left(\mathcal{T}_{i}\right)$ chooses an $N$-dimensional codeword $\mathbf{x}_{i} \in \mathbb{C}^{N},\left\|\mathbf{x}_{i}\right\|^{2} \leq N$, from its codebook, and transmits $\check{\mathbf{x}}_{i}=\sqrt{P_{i}} \mathbf{x}_{i}$ in accordance with its transmit power constraint $\left\|\check{\mathbf{x}}_{i}\right\|^{2} \leq N P_{i}$. In addition, we account for the attenuation of transmit signal $i$ at receiver $j\left(\mathcal{R}_{j}\right)$ through the real-valued coefficients $\eta_{i j}>0$. Defining $\mathbf{y}_{i}$ and $\mathbf{z}_{i} \sim \mathcal{C N}\left(\mathbf{0}, \mathbf{I}_{N}\right)$ as the $N$-dimensional received signal vector and noise vector, respectively, at $\mathcal{R}_{i}$, the input-output relation is given by

$$
\mathbf{y}_{i}=\eta_{i i} h_{i i} \check{\mathbf{x}}_{i}+\eta_{j i} h_{j i} \check{\mathbf{x}}_{j}+\mathbf{z}_{i}
$$

for $i, j=1,2$ and $i \neq j$. Setting $\eta_{11}^{2} P_{1}=\eta_{22}^{2} P_{2}=$ SNR and $\eta_{21}^{2} P_{2}=\eta_{12}^{2} P_{1}=\mathrm{SNR}^{\alpha}$ with $\alpha \in[0, \infty]$ simplifies the exposition and comparison of our results to those in [5] and [10]. The resulting equivalent input-output relation is then given by

$$
\mathbf{y}_{i}=\sqrt{\mathrm{SNR}_{i i}} h_{i}+\sqrt{\mathrm{SNR}^{\alpha}} h_{j i} \mathbf{x}_{j}+\mathbf{z}_{i}
$$


for $i, j=1,2$ and $i \neq j$. We assume that both receivers know the signal-to-noise ratio (SNR) value SNR and the parameter $\alpha$ and $\mathcal{R}_{i}(i=1,2)$ knows $\mathbf{h}_{i}=\left[h_{1 i} h_{2 i}\right]^{T}$ perfectly, whereas the transmitters only know the channel statistics of all channels. The data rate of $\mathcal{T}_{i}$ scales with SNR according to $R_{i}=r_{i} \log \mathrm{SNR}$ where the multiplexing rate $r_{i}$ obeys $0 \leq r_{i} \leq 1$. As a result, for $\mathcal{T}_{i}$ to operate at multiplexing rate $r_{i}$, we need a sequence of codebooks $\mathcal{C}_{i}\left(\mathrm{SNR}, r_{i}\right)$, one for each SNR, with $\left|\mathcal{C}_{i}\left(\mathrm{SNR}, r_{i}\right)\right|=$ $2^{N R_{i}}$ codewords $\left\{\mathbf{x}_{i}^{1}, \mathbf{x}_{i}^{2}, \ldots, \mathbf{x}_{i}^{2^{N R_{i}}}\right\}$. In the following, we will need the multiplexing rate vector $\mathbf{r}=\left[\begin{array}{ll}r_{1} & r_{2}\end{array}\right]^{T}$.

\section{VERY StRong InTERFERENCE}

We call channels with $\alpha \geq 2$ very strong interference channels in the sense of [5]. Throughout this section, we take $N=1$; we will see that this results in optimal performance. In the following, we use the short-hand $x_{i}$ for the first element of the transmit signal vector $\mathbf{x}_{i}, y_{i}$ for the first element of the receive signal vector $\mathbf{y}_{i}$, and $\mathcal{X}_{i}$ for $\mathcal{C}_{i}\left(\mathrm{SNR}, r_{i}\right)$.

The error probability corresponding to ML decoding of $\mathcal{T}_{i}$ at $\mathcal{R}_{i}$ under the assumption that the correctly decoded interference $\mathcal{T}_{j}$ has been removed $(i, j=1,2, j \neq i)$ is denoted by $\mathbb{P}\left[E_{i i} \mid \mathbf{h}_{i}\right]$ We write $\mathbb{P}\left[E_{i j} \mid \mathbf{h}_{j}\right]$ for $i, j=1,2$ and $i \neq j$ for the ML decoding error probability of decoding $\mathcal{T}_{i}$ at receiver $\mathcal{R}_{j}$ under the assumption that $\mathcal{T}_{j}$ is treated as noise. The average (w.r.t. the random channel) ML decoding error probability $\mathbb{E}_{\mathbf{h}_{j}}\left\{\mathbb{P}\left[E_{i j} \mid \mathbf{h}_{j}\right]\right\}$ is denoted by $P\left(E_{i j}\right)$ for $i, j=1,2$. The transmit symbols are assumed equally likely for both transmitters, and hence $\mathbb{P}\left[x_{i}\right]=$ $\frac{1}{\left|\mathcal{X}_{i}\right|}$ for $i=1,2$. The notation $x_{i}^{j} \rightarrow x_{i}^{k}$ represents the event of mistakenly decoding the transmitted codeword $x_{i}^{j} \in \mathcal{X}_{i}$ for the codeword $x_{i}^{k} \in \mathcal{X}_{i}$. Throughout this section, as done in [10], we use the performance metric $P(E)=\max \left\{P\left(E_{11}\right), P\left(E_{22}\right)\right\}$. The DMT realized by a given scheme is then characterized by $d(\mathbf{r})=-\lim _{\mathrm{SNR} \rightarrow \infty} \log [P(E)] / \log \mathrm{SNR}$.

It is shown in [11] that joint decoding of the messages from both transmitters at each receiver achieves the DMT outer bound in [10] given by $d(\mathbf{r}) \leq \min \left\{\left(1-r_{1}\right)^{+},\left(1-r_{2}\right)^{+}\right\}$. In the following, we show that a stripping decoder also achieves this DMT outer bound.

Theorem 1: For the fading IC with I/O relation (2), a stripping decoder yields DMT-optimality, i.e., it realizes

$$
P(E) \doteq \mathrm{SNR}^{-\min \left\{\left(1-r_{1}\right)^{+},\left(1-r_{2}\right)^{+}\right\}}
$$

provided that $\Delta x_{i}=x_{i}^{j}-x_{i}^{k}$ satisfies $\left|\Delta x_{i}\right|^{2} \geq \mathrm{SNR}^{-r_{i}+\epsilon}$ for every pair $x_{i}^{j}, x_{i}^{k}$ in each codebook $\mathcal{X}_{i}, i=1,2$, and for some $\epsilon>0$.

Proof: We start by decoding $\mathcal{T}_{2}$ at $\mathcal{R}_{1}$ while treating $\mathcal{T}_{1}$ as noise, i.e., we have the effective $\mathrm{I} / \mathrm{O}$ relation

$$
y_{1}=\sqrt{\operatorname{SNR}^{\alpha}} h_{21} x_{2}+\tilde{z}
$$

where $\tilde{z}$ is the effective noise term with variance $1+\mathrm{SNR}\left|h_{11}\right|^{2}$. Recall that $h_{11}$ and $h_{21}$ are known at $\mathcal{R}_{1}$ so that we can condition on $h_{11}$. We next note that the worst case (in terms of mutual information and hence outage probability) uncorrelated (with the transmit signal) additive noise under a variance constraint is Gaussian [12, Theorem 1]. In the following, we use the corresponding worst-case outage probability to exponentially upper-bound $P\left(E_{21}\right)$, i.e., we set $\tilde{z} \sim \mathcal{C N}\left(0,1+\mathrm{SNR}\left|h_{11}\right|^{2}\right)$. We start by normalizing the received signal according to

$$
\frac{y_{1}}{\sqrt{1+\mathrm{SNR}\left|h_{11}\right|^{2}}}=\sqrt{\frac{\mathrm{SNR}^{\alpha}}{1+\mathrm{SNR}\left|h_{11}\right|^{2}}} h_{21} x_{2}+z
$$

where $z \sim \mathcal{C N}(0,1)$. We can now upper-bound $\mathbb{P}\left[E_{21} \mid \mathbf{h}_{1}\right]$ as

$$
\begin{aligned}
& \mathbb{P}\left[E_{21} \mid \mathbf{h}_{1}\right]=\sum_{x_{2} \in \mathcal{X}_{2}} \mathbb{P}\left[x_{2}\right] \mathbb{P}\left[E_{21} \mid \mathbf{h}_{1}, x_{2}\right] \\
& \leq\left|\mathcal{X}_{2}\right| \mathbb{P}\left[x_{2}^{\tilde{i}} \rightarrow x_{2}^{\tilde{j}} \mid \mathbf{h}_{1}\right] \\
& \quad \leq\left|\mathcal{X}_{2}\right| \mathrm{Q}\left(\sqrt{\frac{\mathrm{SNR}^{\alpha}\left|h_{21}\right|^{2}\left|\Delta x_{2}\right|^{2}}{2\left(1+\mathrm{SNR}\left|h_{11}\right|^{2}\right)}}\right)
\end{aligned}
$$

where $\left\{x_{2}^{\tilde{i}}, x_{2}^{\tilde{j}}\right\}$ denotes the (or " $\mathrm{a}$ " in the case of multiple pairs with the same distance) pair of symbols with minimum Euclidean distance among all possible pairs of different symbols. We next define the outage event $\mathcal{O}_{i i}$ associated with decoding $\mathcal{T}_{i}$ at $\mathcal{R}_{i}$ $(i=1,2)$ in the absence of interference and its complementary event $\overline{\mathcal{O}}_{i i}$ as follows

$$
\begin{aligned}
& \mathcal{O}_{i i}=\left\{h_{i i}: \log \left(1+\mathrm{SNR}\left|h_{i i}\right|^{2}\right)<R_{i}\right\} \\
& \overline{\mathcal{O}}_{i i}=\left\{h_{i i}: \log \left(1+\mathrm{SNR}\left|h_{i i}\right|^{2}\right) \geq R_{i}\right\}
\end{aligned}
$$

We note that the definitions (9) and (10) are in line with the definitions of $P\left(E_{i i}\right)$ for $i=1,2$. Similarly, we define the event $\mathcal{O}_{i j}$ associated with decoding $\mathcal{T}_{i}$ at $\mathcal{R}_{j}$ while treating $\mathcal{T}_{j}$ as noise $(i, j=1,2$ and $i \neq j)$ and its complementary event $\overline{\mathcal{O}}_{i j}$ as follows

$$
\begin{aligned}
& \mathcal{O}_{i j}=\left\{\mathbf{h}_{j}: \log \left(1+\frac{\mathrm{SNR}^{\alpha}\left|h_{i j}\right|^{2}}{1+\mathrm{SNR}\left|h_{j j}\right|^{2}}\right)<R_{i}\right\} \\
& \overline{\mathcal{O}}_{i j}=\left\{\mathbf{h}_{j}: \log \left(1+\frac{\mathrm{SNR}^{\alpha}\left|h_{i j}\right|^{2}}{1+\mathrm{SNR}\left|h_{j j}\right|^{2}}\right) \geq R_{i}\right\} .
\end{aligned}
$$

Next, we upper-bound $P\left(E_{21}\right)$ according to

$$
\begin{aligned}
& P\left(E_{21}\right)=\mathbb{E}_{\mathbf{h}_{1}}\left\{\mathbb{P}\left[E_{21} \mid \mathbf{h}_{1}\right]\right\}= \\
& \mathbb{E}_{\mathbf{h}_{1}}\left\{\mathbb{P}\left[\mathcal{O}_{21}\right] \mathbb{P}\left[E_{21} \mid \mathbf{h}_{1}, \mathcal{O}_{21}\right]+\mathbb{P}\left[\overline{\mathcal{O}}_{21}\right] \mathbb{P}\left[E_{21} \mid \mathbf{h}_{1}, \overline{\mathcal{O}}_{21}\right]\right\} \\
& \quad \leq \mathbb{P}\left[\mathcal{O}_{21}\right]+\mathbb{E}_{\mathbf{h}_{1}}\left\{\mathbb{P}\left[E_{21} \mid \mathbf{h}_{1}, \overline{\mathcal{O}}_{21}\right]\right\} \\
& \quad \leq \mathbb{P}\left[\mathcal{O}_{21}\right]+\operatorname{SNR}^{r_{2}} \mathrm{Q}\left(\sqrt{\frac{\mathrm{SNR}^{r_{2}}\left|\Delta x_{2}\right|^{2}}{2}}\right)
\end{aligned}
$$

where (11) follows from Bayes's rule and (12) is obtained by upper-bounding $\mathbb{P}\left[E_{21} \mid \mathbf{h}_{1}, \mathcal{O}_{21}\right]$ and $\mathbb{P}\left[\overline{\mathcal{O}}_{21}\right]$ by 1 . Finally, follows by using the fact that $\overline{\mathcal{O}}_{21}$ implies $\frac{\mathrm{SNR}^{\alpha}\left|h_{21}\right|^{2}}{1+\mathrm{SNR}\left|h_{11}\right|^{2}} \geq 2^{R_{2}}-1$, and invoking $R_{2}=r_{2} \log \mathrm{SNR},\left|\mathcal{X}_{2}\right|=\mathrm{SNR}^{r_{2}}$, and SNR $\gg 1$ in 8). It can be shown that $\mathbb{P}\left[\mathcal{O}_{21}\right] \doteq \mathrm{SNR}^{-\left(\alpha-1-r_{2}\right)^{+}}$for $\alpha \geq 2$ [10]. Further, since $\left|\Delta x_{2}\right|^{2} \geq \mathrm{SNR}^{-r_{2}+\epsilon}$, for $\epsilon>0$, by assumption, we can further simplify the above as the second term in 13 decays exponentially in SNR whereas the first term decays polynomially, i.e., $\mathbb{E}_{\mathbf{h}_{1}}\left\{\mathbb{P}\left[E_{21} \mid \mathbf{h}_{1}\right]\right\} \leq \mathbb{P}\left[\mathcal{O}_{21}\right] \doteq \mathrm{SNR}^{-\left(\alpha-1-r_{2}\right)^{+}}$. We proceed to analyze decoding of $\mathcal{T}_{1}$ at $\mathcal{R}_{1}$ and start by defining $\bar{x}_{2}$ as the result of decoding $\mathcal{T}_{2}$ at $\mathcal{R}_{1}$. Note that we do not need 
to assume that $\mathcal{T}_{2}$ was decoded correctly at $\mathcal{R}_{1}$. We begin by upper-bounding $\mathbb{P}\left[E_{11} \mid \mathbf{h}_{1}\right]$ given $\bar{x}_{2}$ :

$$
\begin{aligned}
& \mathbb{P}\left[E_{11} \mid \mathbf{h}_{1}, \bar{x}_{2}\right]=\sum_{x_{1} \in \mathcal{X}_{1}} \sum_{x_{2} \in \mathcal{X}_{2}} \mathbb{P}\left[x_{1}\right] \mathbb{P}\left[x_{2}\right] \mathbb{P}\left[E_{1} \mid \mathbf{h}_{1}, x_{1}, x_{2}, \bar{x}_{2}\right] \\
& \leq \frac{\left|\mathcal{X}_{1}\right|}{\left|\mathcal{X}_{2}\right|} \sum_{k=1}^{\left|\mathcal{X}_{2}\right|} \mathbb{P}\left[x_{1}^{\tilde{i}} \rightarrow x_{1}^{\tilde{j}} \mid \mathbf{h}_{1}, x_{2}^{k}, \bar{x}_{2}\right]
\end{aligned}
$$

where $\left\{x_{1}^{\tilde{i}}, x_{1}^{\tilde{j}}\right\}$ denotes the (or "a" in the case of multiple pairs with the same distance) pair of symbols with minimum Euclidean distance among all possible pairs of different symbols. Next, we further upper-bound $\mathbb{P}\left[E_{11} \mid \mathbf{h}_{1}, \bar{x}_{2}\right]$ by considering two events; namely, when $\mathcal{R}_{1}$ decodes $\mathcal{T}_{2}$ correctly and when it does not:

$$
\begin{aligned}
& \mathbb{P}\left[E_{11} \mid \mathbf{h}_{1}, \bar{x}_{2}\right] \leq \\
& \frac{\left|\mathcal{X}_{1}\right|}{\left|\mathcal{X}_{2}\right|} \sum_{k=1}^{\left|\mathcal{X}_{2}\right|}\left(\mathbb{P}\left[\bar{x}_{2}=x_{2}^{k} \mid \mathbf{h}_{1}, x_{2}^{k}\right] \mathbb{P}\left[x_{1}^{\tilde{i}} \rightarrow x_{1}^{\tilde{j}} \mid \mathbf{h}_{1}, x_{2}^{k}, \bar{x}_{2}, \bar{x}_{2}=x_{2}^{k}\right]\right. \\
& \left.+\mathbb{P}\left[\bar{x}_{2} \neq x_{2}^{k} \mid \mathbf{h}_{1}, x_{2}^{k}\right] \mathbb{P}\left[x_{1}^{\tilde{i}} \rightarrow x_{1}^{\tilde{j}} \mid \mathbf{h}_{1}, x_{2}^{k}, \bar{x}_{2}, \bar{x}_{2} \neq x_{2}^{k}\right]\right),
\end{aligned}
$$

where $\mathbb{P}\left[x_{1}^{\tilde{i}} \rightarrow x_{1}^{\tilde{j}} \mid \mathbf{h}_{1}, x_{2}^{k}, \bar{x}_{2}, \bar{x}_{2}=x_{2}^{k}\right]$ is the probability of mistakenly decoding $x_{1}^{\tilde{i}}$ for $x_{1}^{\tilde{j}}$ given that $\mathcal{T}_{2}$ transmitted $x_{2}^{k}$ and $\mathcal{R}_{1}$ decoded $\mathcal{T}_{2}$ correctly, i.e., $\bar{x}_{2}=x_{2}^{k}$. The quantity $\mathbb{P}\left[\bar{x}_{2}=x_{2}^{k} \mid \mathbf{h}_{1}, x_{2}^{k}\right]$ is the probability of decoding $\mathcal{T}_{2}$ correctly given that $x_{2}^{k}$ was transmitted. By upper-bounding $\mathbb{P}\left[\bar{x}_{2}=x_{2}^{k} \mid \mathbf{h}_{1}, x_{2}^{k}\right]$ and $\mathbb{P}\left[x_{1}^{\tilde{i}} \rightarrow x_{1}^{\tilde{j}} \mid \mathbf{h}_{1}, x_{2}^{k}, \bar{x}_{2}, \bar{x}_{2} \neq x_{2}^{k}\right]$ in (15) by 1 , we arrive at

$$
\begin{gathered}
\mathbb{P}\left[E_{11} \mid \mathbf{h}_{1}, \bar{x}_{2}\right] \leq \frac{\left|\mathcal{X}_{1}\right|}{\left|\mathcal{X}_{2}\right|} \sum_{k=1}^{\left|\mathcal{X}_{2}\right|} \mathbb{P}\left[x_{1}^{i} \rightarrow x_{1}^{\tilde{j}} \mid \mathbf{h}_{1}, x_{2}^{k}, \bar{x}_{2}, \bar{x}_{2}=x_{2}^{k}\right]+ \\
\frac{\left|\mathcal{X}_{1}\right|}{\left|\mathcal{X}_{2}\right|} \sum_{k=1}^{\left|\mathcal{X}_{2}\right|} \mathbb{P}\left[\bar{x}_{2} \neq x_{2}^{k} \mid \mathbf{h}_{1}, x_{2}^{k}\right]
\end{gathered}
$$

Next, noting that $\frac{1}{\left|\mathcal{X}_{2}\right|} \sum_{k=1}^{\left|\mathcal{X}_{2}\right|} \mathbb{P}\left[\bar{x}_{2} \neq x_{2}^{k} \mid \mathbf{h}_{1}, x_{2}^{k}\right] \leq \mathbb{P}\left[E_{21} \mid \mathbf{h}_{1}\right]$ and invoking the corresponding upper bound $(8)$ in $(16)$, we get

$$
\begin{aligned}
\mathbb{P}\left[E_{11} \mid \mathbf{h}_{1}, \bar{x}_{2}\right] \leq & \left|\mathcal{X}_{1}\right| \mathrm{Q}\left(\sqrt{\frac{\mathrm{SNR}\left|h_{11}\right|^{2}\left|\Delta x_{1}\right|^{2}}{2}}\right)+ \\
& \left|\mathcal{X}_{1}\right|\left|\mathcal{X}_{2}\right| \mathrm{Q}\left(\sqrt{\frac{\mathrm{SNR}^{\alpha}\left|h_{21}\right|^{2}\left|\Delta x_{2}\right|^{2}}{2\left(1+\mathrm{SNR}\left|h_{11}\right|^{2}\right)}}\right) .
\end{aligned}
$$

The first term on the RHS of (17) follows from the first term on the RHS of (16), since given $\bar{x}_{2}=x_{2}^{k}$, the interference is subtracted out perfectly, leaving an effective SISO channel without interference. We are now in a position to upper-bound $P\left(E_{11}\right)$ :

$$
\begin{aligned}
& P\left(E_{11}\right)=\mathbb{E}_{\mathbf{h}_{1}}\left\{\mathbb{P}\left[E_{11} \mid \mathbf{h}_{1}\right]\right\} \leq \mathbb{E}_{\mathbf{h}_{1}}\left\{\mathbb{P}\left[E_{11} \mid \mathbf{h}_{1}, \bar{x}_{2}\right]\right\} \\
& \leq \mathbb{E}_{\mathbf{h}_{1}}\left\{\left|\mathcal{X}_{1}\right| \mathrm{Q}\left(\sqrt{\frac{\mathrm{SNR}\left|h_{11}\right|^{2}\left|\Delta x_{1}\right|^{2}}{2}}\right)\right\}+ \\
& \mathbb{E}_{\mathbf{h}_{1}}\left\{\left|\mathcal{X}_{1}\right|\left|\mathcal{X}_{2}\right| \mathrm{Q}\left(\sqrt{\frac{\mathrm{SNR}^{\alpha}\left|h_{21}\right|^{2}\left|\Delta x_{2}\right|^{2}}{2\left(1+\mathrm{SNR}\left|h_{11}\right|^{2}\right)}}\right)\right\} .
\end{aligned}
$$

Here, 18 follows since the error probability incurred by using the stripping decoder constitutes a natural upper bound on $\mathbb{E}_{\mathbf{h}_{1}}\left\{\mathbb{P}\left[E_{11} \mid \mathbf{h}_{1}\right]\right\}$. We upper-bound $(19)$ by splitting each of the two terms into outage and no outage sets using Bayes's rule to arrive at

$$
\begin{aligned}
& P\left(E_{11}\right)=\mathbb{E}_{\mathbf{h}_{1}\left\{\mathbb{P}\left[E_{11} \mid \mathbf{h}_{1}\right]\right\} \leq} \\
& \mathbb{P}\left[\mathcal{O}_{11}\right]+\mathrm{SNR}^{r_{1}} \mathrm{Q}\left(\sqrt{\frac{\mathrm{SNR}^{r_{1}}\left|\Delta x_{1}\right|^{2}}{2}}\right)+\mathbb{P}\left[\mathcal{O}_{21}\right]+ \\
& \operatorname{SNR}^{r_{1}+r_{2}} \mathrm{Q}\left(\sqrt{\frac{\mathrm{SNR}^{r_{2}}\left|\Delta x_{2}\right|^{2}}{2}}\right) .
\end{aligned}
$$

The second and fourth terms on the RHS of (20) follow from (19) since $\overline{\mathcal{O}}_{11}$ and $\overline{\mathcal{O}}_{21}$ imply SNR $\left|h_{11}\right|^{2} \geq 2^{R_{1}}-1$ and $\frac{\mathrm{SNR}^{\alpha}\left|h_{21}\right|^{2}}{1+\mathrm{SNR}\left|h_{11}\right|^{2}} \geq 2^{R_{2}}-1$, respectively, and since $R_{i}=r_{i} \log \mathrm{SNR}$ $\left|\mathcal{X}_{i}\right|=\mathrm{SNR}^{r_{i}}$ for $i=1,2$ and $\mathrm{SNR} \gg 1$. Given that the minimum Euclidean distances in each codebook, $\left|\Delta x_{1}\right|^{2}$ and $\left|\Delta x_{2}\right|^{2}$, obey $\left|\Delta x_{1}\right|^{2} \geq \mathrm{SNR}^{-r_{1}+\epsilon}$ and $\left|\Delta x_{2}\right|^{2} \geq \mathrm{SNR}^{-r_{2}+\epsilon}$, for some $\epsilon>0$, by assumption, we get

$$
\begin{aligned}
& P\left(E_{11}\right)=\mathbb{E}_{\mathbf{h}_{1}}\left\{\mathbb{P}\left[E_{11} \mid \mathbf{h}_{1}\right]\right\} \leq \mathbb{P}\left[\mathcal{O}_{11}\right]+\mathbb{P}\left[\mathcal{O}_{21}\right] \\
& \doteq \mathrm{SNR}^{-\left(1-r_{1}\right)^{+}}+\mathrm{SNR}^{-\left(\alpha-1-r_{2}\right)^{+}} \\
& \doteq \mathrm{SNR}^{-\min \left\{\left(1-r_{1}\right)^{+},\left(\alpha-1-r_{2}\right)^{+}\right\}} \text {. }
\end{aligned}
$$

Similar derivations for decoding at $\mathcal{R}_{2}$ lead to $P\left(E_{22}\right) \leq \mathrm{SNR}^{-\min }\left\{\left(1-r_{2}\right)^{+},\left(\alpha-1-r_{1}\right)^{+}\right\}$. We note that the error probability of decoding $\mathcal{T}_{i}$ at $\mathcal{R}_{i}$ is exponentially lower-bounded by $\mathbb{P}\left[\mathcal{O}_{i i}\right]$ for $i=1,2$ [13]. Hence, $P\left(E_{i i}\right)$ is sandwiched according to

$$
\mathrm{SNR}^{-\left(1-r_{i}\right)^{+}} \dot{\leq} P\left(E_{i i}\right) \dot{\leq} \mathrm{SNR}^{-\min \left\{\left(1-r_{i}\right)^{+},\left(\alpha-1-r_{j}\right)^{+}\right\}}
$$

for $i, j=1,2$ and $i \neq j$. The proof is concluded by first upperbounding $P(E)=\max \left\{P\left(E_{11}\right), P\left(E_{22}\right)\right\}$ as

$$
\begin{aligned}
& P(E) \dot{\max }\left\{\mathrm{SNR}^{-\min \left\{\left(1-r_{1}\right)^{+},\left(\alpha-1-r_{2}\right)^{+}\right\}},\right. \\
&\left.\mathrm{SNR}^{-\min \left\{\left(1-r_{2}\right)^{+},\left(\alpha-1-r_{1}\right)^{+}\right\}}\right\} \\
& \doteq \mathrm{SNR}^{-\min \left\{\left(1-r_{1}\right)^{+},\left(1-r_{2}\right)^{+}\right\}}
\end{aligned}
$$

where 25] is a consequence of the assumption $\alpha \geq 2$. Secondly, $P(E)$ can be lower-bounded using the outage bounds on the individual error probabilities $P\left(E_{11}\right)$ and $P\left(E_{22}\right)$ :

$$
\mathrm{SNR}^{-\min \left\{\left(1-r_{1}\right)^{+},\left(1-r_{2}\right)^{+}\right\}} \dot{\leq} P(E) .
$$

Since the SNR exponents in the upper bound 25 and the lower bound 26 match, we can conclude that $P(E) \doteq$ $\mathrm{SNR}^{-\min \left\{\left(1-r_{1}\right)^{+},\left(1-r_{2}\right)^{+}\right\}}$which establishes the desired result.

Remark 1: We can immediately conclude from Theorem 1 that using a sequence of codebooks that is DMT-optimal for the SISO channel for both users results in DMT-optimality for the IC under very strong interference.

Remark 2: If $R_{1}=R_{2}=r \log$ SNR and we use sequences of codebooks $\mathcal{C}(\mathrm{SNR}, r)$ satisfying the conditions of Theorem 1 for both users, then we have $P\left(E_{11}\right) \doteq P\left(E_{22}\right) \doteq \mathrm{SNR}^{-(1-r)^{+}}$ as a simple consequence of (24). This means that in the special case, where each $\mathcal{T}_{i}$ transmits at the same multiplexing rate $r$, 
we have the stronger result that the single user DMT, i.e., the DMT that is achievable for a SISO channel in the absence of any interferers, is achievable for both users. In effect, under very strong interference and when the two users operate at the same multiplexing rate, the interference channel effectively gets decoupled. For $r_{1} \neq r_{2}$, we can, in general, not arrive at the same conclusion as the SNR exponents in 24) do not necessarily match. Joint decoding at both receivers is, however, shown in [11] to decouple the very strong interference (fading) channel for $\alpha \geq 2$ for all values of $r_{i}, i=1,2$, i.e., for $0 \leq r_{i} \leq 1$, $i=1,2$.

\section{General Interference Channels AND HAN AND KOBAYASHI SCHEMES}

The HK rate region [14] remains the best known achievable rate region for the Gaussian IC [3], [15]. The original HK strategy lets each transmitter split its message into two messages, and allows each receiver to decode part of the interfering signal.

In the following, we analyze the DMT of a superposition HK scheme where $\mathcal{T}_{i}$ transmits the $N$-dimensional $(N \geq 2)$ vector $\mathbf{x}_{i}=\mathbf{u}_{i}+\mathbf{w}_{i}$ with $\mathbf{u}_{i}$ and $\mathbf{w}_{i}$ representing the private and the public message, respectively. All assumptions of Section I remain valid, and we allow all levels of interference, i.e., $\alpha \geq 0$. The power constraints for $\mathbf{u}_{i}$ and $\mathbf{w}_{i}$ are

$$
\left\|\mathbf{u}_{i}\right\| \leq \sqrt{\frac{N}{\mathrm{SNR}^{1-p_{i}}}}, \quad\left\|\mathbf{w}_{i}\right\| \leq \sqrt{N}\left(1-\sqrt{\frac{1}{\mathrm{SNR}^{1-p_{i}}}}\right)
$$

so that $\left\|\mathbf{x}_{i}\right\| \leq\left\|\mathbf{u}_{i}\right\|+\left\|\mathbf{w}_{i}\right\|=\sqrt{N}$. Here, $0 \leq p_{i}<1$ accounts for the exponential order of the power allocated to the private message. The power split is assumed fixed and is independent of the channel realizations. When both the private and the public message are allocated maximum power, we have $\frac{\left\|\mathbf{w}_{i}\right\|^{2}}{\left\|\mathbf{u}_{i}\right\|^{2}} \doteq \mathrm{SNR}^{1-p_{i}}$. We emphasize that any $p_{i}<1$ constitutes a valid power split. We explain in [11] why we can omit all the cases with $p_{i}<0$ except for $p_{i}=-\infty$. The special case $p_{i}=-\infty$ corresponds to using public messages only and is, therefore, similar to a multiuser setup. This case is treated separately in the following and is referred to by the subscript $M U$.

We assume that $\mathcal{T}_{i}$ transmits at rate $R_{i}=r_{i} \log$ SNR where the rates for the private and the public messages, respectively, are $S_{i}=s_{i} \log$ SNR and $T_{i}=t_{i} \log$ SNR with $r_{i}=s_{i}+t_{i}$, $s_{i}, t_{i} \geq 0$, and $0 \leq r_{i} \leq 1$. The codebooks corresponding to the private and the public message parts are denoted as $\mathcal{C}^{\mathbf{u}_{i}}\left(\mathrm{SNR}, s_{i}\right)$ and $\mathcal{C}^{\mathbf{w}_{i}}\left(\mathrm{SNR}, t_{i}\right)$, respectively, and satisfy $\left|\mathcal{C}^{\mathbf{u}_{i}}\left(\mathrm{SNR}, s_{i}\right)\right|=$ $\mathrm{SNR}^{N s_{i}}$ and $\left|\mathcal{C}^{\mathbf{w}_{i}}\left(\mathrm{SNR}, t_{i}\right)\right|=\mathrm{SNR}^{N t_{i}}$. Clearly, $\mathcal{C}^{\mathbf{x}_{i}}\left(\mathrm{SNR}, r_{i}\right)=$ $\mathcal{C}^{\mathbf{u}_{i}}\left(\mathrm{SNR}, s_{i}\right) \times \mathcal{C}^{\mathbf{w}_{i}}\left(\mathrm{SNR}, t_{i}\right)$ with $\left|\mathcal{C}^{\mathbf{x}_{i}}\left(\mathrm{SNR}, r_{i}\right)\right|=\mathrm{SNR}^{r_{i}}$. In the following, we will need the private message multiplexing rate vector $\mathbf{s}=\left[\begin{array}{ll}s_{1} & s_{2}\end{array}\right]^{T}$ and the SNR exponent vector $\mathbf{p}=\left[\begin{array}{ll}p_{1} & p_{2}\end{array}\right]^{T}$ of the private messages. As before, our performance metric is $P(E)=\max \left\{P\left(E_{11}\right), P\left(E_{22}\right)\right\}$.

Theorem 2: The maximum DMT achievable by a fixedpower-split HK scheme is given by

$$
d(\mathbf{r})=\max \left\{d_{H K}(\mathbf{r}), d_{M U}(\mathbf{r})\right\}
$$

where $d_{M U}(\mathbf{r})=\min _{i=1,2,3}\left\{d_{M U}^{i}(\mathbf{r})\right\}$ with

$$
\begin{aligned}
& d_{M U}^{i}(\mathbf{r})=\left(1-r_{i}\right)^{+} \text {for } i=1,2 \\
& d_{M U}^{3}(\mathbf{r})=\left(1-r_{1}-r_{2}\right)^{+}+\left(\alpha-r_{1}-r_{2}\right)^{+}
\end{aligned}
$$

and

$$
d_{H K}(\mathbf{r})=\max _{\mathbf{s}, \mathbf{p}} d(\mathbf{r}, \mathbf{s}, \mathbf{p})
$$

where the optimization is carried out subject to the constraints $s_{i}+t_{i}=r_{i}$ with $s_{i}, t_{i} \geq 0$, and $0 \leq p_{i}<1$, all for $i=1,2$, and

$$
\begin{aligned}
& d(\mathbf{r}, \mathbf{s}, \mathbf{p})=\min _{\substack{k=1,2 \\
l=1,2, \ldots, 6}}\left\{d_{k l}(\mathbf{r}, \mathbf{s}, \mathbf{p})\right\} \\
& d_{i 1}(\mathbf{r}, \mathbf{s}, \mathbf{p})= \begin{cases}\left(p_{i}-s_{i}\right)^{+}, & \text {if } p_{j}<1-\alpha \\
\left(1-\alpha-p_{j}+p_{i}-s_{i}\right)^{+}, & \text {if } p_{j} \geq 1-\alpha\end{cases} \\
& d_{i 2}(\mathbf{r}, \mathbf{s}, \mathbf{p})= \begin{cases}\left(1-r_{i}+s_{i}\right)^{+}, & \text {if } p_{j}<1-\alpha \\
\left(2-\alpha-p_{j}-r_{i}+s_{i}\right)^{+}, & \text {if } p_{j} \geq 1-\alpha\end{cases} \\
& d_{i 3}(\mathbf{r}, \mathbf{s}, \mathbf{p})= \begin{cases}\left(1-r_{i}\right)^{+}, & \text {if } p_{j}<1-\alpha \\
\left(2-\alpha-p_{j}-r_{i}\right)^{+}, & \text {if } p_{j} \geq 1-\alpha\end{cases} \\
& d_{i 4}(\mathbf{r}, \mathbf{s}, \mathbf{p})=\left\{\begin{array}{c}
\left(p_{i}-s_{i}-r_{j}+s_{j}\right)^{+}+\left(\alpha-s_{i}-r_{j}+s_{j}\right)^{+}, \\
\quad \text { if } p_{j}<1-s_{i}-r_{j}+s_{j} \\
\left(p_{i}-s_{i}-r_{j}+s_{j}\right)^{+}, \\
\quad \text { if } p_{j} \geq 1-s_{i}-r_{j}+s_{j} \text { and } p_{j}<1-\alpha \\
\left(1-\alpha-p_{j}+p_{i}-s_{i}-r_{j}+s_{j}\right)^{+}, \\
\quad \text { if } p_{j} \geq 1-s_{i}-r_{j}+s_{j} \text { and } p_{j} \geq 1-\alpha
\end{array}\right. \\
& d_{i 5}(\mathbf{r}, \mathbf{s}, \mathbf{p})=\left\{\begin{array}{l}
\left(1-\sum_{k=1}^{2} r_{k}+\sum_{l=1}^{2} s_{l}\right)^{+}+\left(\alpha-\sum_{k=1}^{2} r_{k}+\sum_{l=1}^{2} s_{l}\right)^{+} \\
\text {if } p_{j}<1-\sum_{k=1}^{2} r_{k}+\sum_{l=1}^{2} s_{l}, \\
\left(1-\sum_{k=1}^{2} r_{k}+\sum_{l=1}^{2} s_{l}\right)^{+}, \\
\text {if } p_{j} \geq 1-\sum_{k=1}^{2} r_{k}+\sum_{l=1}^{2} s_{l} \text { and } p_{j}<1-\alpha \\
\left(2-\alpha-p_{j}-\sum_{k=1}^{2} r_{k}+\sum_{l=1}^{2} s_{l}\right)^{+}, \\
\text {if } p_{j} \geq 1-\sum_{k=1}^{2} r_{k}+\sum_{l=1}^{2} s_{l} \text { and } p_{j} \geq 1-\alpha
\end{array}\right. \\
& d_{i 6}(\mathbf{r}, \mathbf{s}, \mathbf{p})=\left\{\begin{array}{c}
\left(1-r_{i}-r_{j}+s_{j}\right)^{+}+\left(\alpha-r_{i}-r_{j}+s_{j}\right)^{+}, \\
\quad \text { if } p_{j}<1-r_{i}-r_{j}+s_{j} \\
\left(1-r_{i}-r_{j}+s_{j}\right)^{+}, \\
\quad \text { if } p_{j} \geq 1-r_{i}-r_{j}+s_{j} \text { and } p_{j}<1-\alpha \\
\left(2-\alpha-p_{j}-r_{i}-r_{j}+s_{j}\right)^{+}, \\
\quad \text { if } p_{j} \geq 1-r_{i}-r_{j}+s_{j} \text { and } p_{j} \geq 1-\alpha
\end{array}\right.
\end{aligned}
$$

with $i, j=1,2$ and $i \neq j$.

We shall next provide code design criteria for achieving the DMT in Theorem 2.

Theorem 3: For a given rate tuple r, either $d_{H K}(\mathbf{r})$ or $d_{M U}(\mathbf{r})$ dominates in Theorem 2.

i) If $d_{H K}(\mathbf{r}) \leq d_{M U}(\mathbf{r})$, the DMT in Theorem 2 is achieved as follows. Denote $j^{*}=\arg \min _{i=1,2,3} d_{M U}^{i}(\mathbf{r})$. Let 
$\Gamma_{i}(\mathbf{r})=\left[\gamma_{i}^{1}(\mathbf{r}) \gamma_{i}^{2}(\mathbf{r})\right]^{T}$ be the functions such that $d_{M U}^{j^{*}}(\mathbf{r})=d_{M U}^{i}\left(\Gamma_{i}(\mathbf{r})\right)$ for $i=1,2,3$. Then, the DMT in Theorem 2 is achieved by employing a sequence (in SNR) of codebooks satisfying

$$
\begin{aligned}
& \left\|\Delta \mathbf{x}_{i}\right\|^{2} \geq \mathrm{SNR}^{-\gamma_{i}^{i}(\mathbf{r})+\epsilon}, \\
& \lambda_{\min }\left(\Delta \mathbf{X}(\Delta \mathbf{X})^{H}\right) \geq \mathrm{SNR}^{-\gamma_{3}^{1}(\mathbf{r})-\gamma_{3}^{2}(\mathbf{r})+\epsilon}
\end{aligned}
$$

for some $1^{1} \epsilon>0$, where $\Delta \mathbf{x}_{i}=\hat{\mathbf{x}}_{i}-\tilde{\mathbf{x}}_{i}, i=1,2$, with $\hat{\mathbf{x}}_{i}, \tilde{\mathbf{x}}_{i} \in \mathcal{C}^{\mathbf{x}_{i}}\left(\mathrm{SNR}, r_{i}\right)$, and $\Delta \mathbf{X}=\left[\Delta \mathbf{x}_{1} \Delta \mathbf{x}_{2}\right]$.

ii) If $d_{H K}(\mathbf{r})>d_{M U}(\mathbf{r})$, then define the codeword difference vectors $\Delta \mathbf{u}_{i}=\sqrt{\operatorname{SNR}^{1-p_{i}}}\left(\tilde{\mathbf{u}}_{i}-\hat{\mathbf{u}}_{i}\right), \Delta \mathbf{w}_{i}=$ $\tilde{\mathbf{w}}_{i}-\hat{\mathbf{w}}_{i}$, and $\Delta \mathbf{x}_{i}=\tilde{\mathbf{x}}_{i}-\hat{\mathbf{x}}_{i}$ with $\tilde{\mathbf{u}}_{i}, \hat{\mathbf{u}}_{i} \in \mathcal{C}^{\mathbf{u}_{i}}\left(\mathrm{SNR}, s_{i}\right)$, $\tilde{\mathbf{w}}_{i}, \hat{\mathbf{w}}_{i} \in \mathcal{C}^{\mathbf{w}_{i}}\left(\mathrm{SNR}, t_{i}\right)$ and $\tilde{\mathbf{x}}_{i}, \hat{\mathbf{x}}_{i} \in \mathcal{C}^{\mathbf{x}_{i}}\left(\mathrm{SNR}, r_{i}\right)$, for $i=1,2$. Further, define $\Delta \mathbf{A}_{i j}=\left[\Delta \mathbf{u}_{i} \Delta \mathbf{w}_{j}\right], \Delta \mathbf{B}_{i j}=$ $\left[\Delta \mathbf{w}_{i} \Delta \mathbf{w}_{j}\right]$, and $\Delta \mathbf{C}_{i j}=\left[\Delta \mathbf{x}_{i} \Delta \mathbf{w}_{j}\right]$ for $i, j=1,2$ and $i \neq j$. Denote the optimizing values of $\mathbf{s}$, $\mathbf{t}$, and $\mathbf{p}$ obtained by solving (29) as $\mathbf{s}^{*}, \mathbf{t}^{*}$, and $\mathbf{p}^{*}$, respectively. We let

$$
\left[k^{*} l^{*}\right]=\underset{\substack{k=1,2 \\ l=1,2,3,4,5,6}}{\arg \min }\left(d_{k l}(\mathbf{r}, \mathbf{s}, \mathbf{p})\right) .
$$

Further, let the functions $\Upsilon_{n m}(\mathbf{r})=\left[v_{n m}^{1}(\mathbf{r}) v_{n m}^{2}(\mathbf{r})\right]^{T}$ and $\Psi_{n m}\left(\mathbf{s}^{*}\right)=\left[\psi_{n m}^{1}\left(\mathbf{s}^{*}\right) \psi_{n m}^{2}\left(\mathbf{s}^{*}\right)\right]^{T}$ be such that

$$
d_{k^{*} l^{*}}\left(\mathbf{r}, \mathbf{s}^{*}, \mathbf{p}^{*}\right)=d_{n m}\left(\Upsilon_{n m}(\mathbf{r}), \Psi_{n m}\left(\mathbf{s}^{*}\right), \mathbf{p}^{*}\right)
$$

for all $n=1,2$ and $m=1,2, \ldots, 6$. Then, the DMT in Theorem 2 is achieved by employing a sequence (in SNR) of codebooks satisfying

$$
\begin{aligned}
& \left\|\Delta \mathbf{u}_{i}\right\|^{2} \geq \mathrm{SNR}^{-\psi_{i 1}^{i}\left(\mathbf{s}^{*}\right)+\epsilon} \\
& \left\|\Delta \mathbf{w}_{i}\right\|^{2} \geq \mathrm{SNR}^{-v_{i 2}^{i}(\mathbf{r})+\psi_{i 2}^{i}\left(\mathbf{s}^{*}\right)+\epsilon} \\
& \left\|\Delta \mathbf{x}_{i}\right\|^{2} \geq \mathrm{SNR}^{-v_{i 3}^{i}(\mathbf{r})+\epsilon} \\
& \lambda_{\min }\left(\Delta \mathbf{A}_{i j}\left(\Delta \mathbf{A}_{i j}\right)^{H}\right) \geq \mathrm{SNR}^{-\psi_{i 4}^{i}\left(\mathbf{s}^{*}\right)-v_{j 4}^{j}(\mathbf{r})+\psi_{j 4}^{j}\left(\mathbf{s}^{*}\right)+\epsilon} \\
& \lambda_{\min }\left(\Delta \mathbf{B}_{i j}\left(\Delta \mathbf{B}_{i j}\right)^{H}\right) \geq \mathrm{SNR}-\sum_{k=1}^{2} v_{k 5}^{k}(\mathbf{r})+\sum_{j=1}^{2} \psi_{j 5}^{j}\left(\mathbf{s}^{*}\right)+\epsilon \\
& \lambda_{\min }\left(\Delta \mathbf{C}_{i j}\left(\Delta \mathbf{C}_{i j}\right)^{H}\right) \geq \mathrm{SNR}^{-v_{i 6}^{i}(\mathbf{r})-v_{j 6}^{j}(\mathbf{r})+\psi_{j 6}^{j}\left(\mathbf{s}^{*}\right)+\epsilon}
\end{aligned}
$$

for every pair of codewords in each codebook for $i, j=$ $1,2, i \neq j$, and for some ${ }^{1} \epsilon>0$.

For a proof of Theorems 2 and 3, we refer to [11].

Remark 3: Whenever $d_{H K}(\mathbf{r}) \leq d_{M U}(\mathbf{r})$ and $\alpha=1$, the code design criteria in Theorem 3 (stated in (30) and (31)) are equivalent to the criteria for achieving the optimal DMT in a multiple access channel (MAC). The existence of DMToptimal codes for the MAC is shown in [16] and an explicit construction [17] is shown to be DMT-optimal in [18]. For $d_{H K}(\mathbf{r})>d_{M U}(\mathbf{r})$, it is an open question whether there are superposition codes that satisfy (33) and hence, achievability of the DMT through the fixed-power-split superposition HK scheme depends on whether this question can be resolved positively.

Numerical result: For $\alpha=2 / 3$ and $r_{1}=r_{2}=r$, Fig. 1 shows the DMT achieved by the fixed-power-split HK scheme (HK) in comparison to the outer bound in [10] (AL08), to joint decoding (JD), to treating interference as noise (TIAN), and to time-sharing (TS). It is shown in [11] that the outer bound AL08

\footnotetext{
${ }^{1}$ We note that all $\epsilon$ 's in $30,-31$ and equation block 33 can be different.
}

is loose under moderate interference, i.e., when $2 / 3 \leq \alpha<1$, and that the HK scheme is DMT-optimal in this range.

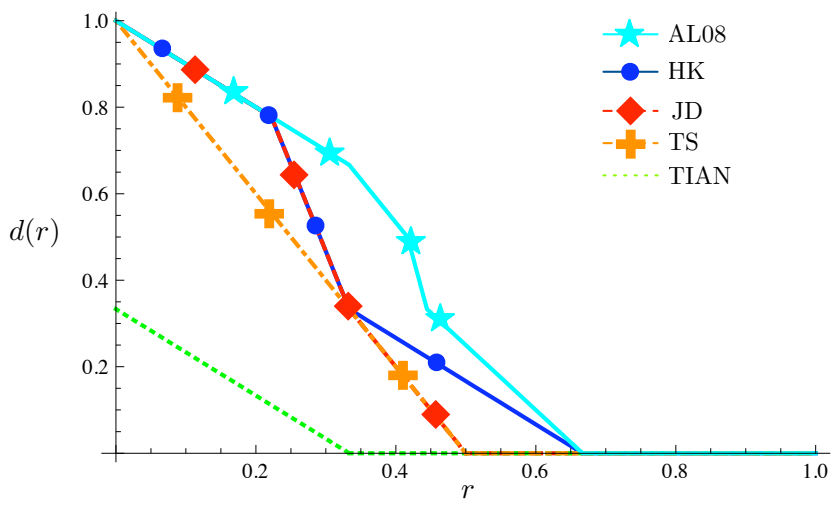

Fig. 1. Symmetric rate DMT for $\alpha=2 / 3$ and for various schemes.

\section{REFERENCES}

[1] H. Sato, "The capacity of the gaussian interference channel under strong interference," IEEE Trans. Inf. Theory, vol. 27, no. 6, pp. 786-788, Nov. 1981.

[2] A. Carleial, "A case where interference does not reduce capacity," IEEE Trans. Inf. Theory, vol. 21, no. 5, pp. 569-570, Sep. 1975.

[3] I. Sason, "On achievable rate regions for the Gaussian interference channel," IEEE Trans. Inf. Theory, vol. 50, no. 6, pp. 1345-1356, Jun. 2004.

[4] R. Etkin, D. N. C. Tse, and H. Wang, "Gaussian interference channel capacity to within one bit: The symmetric case," in Proc. IEEE Inf. Theory Workshop (ITW), Oct. 2006, pp. 601-605.

[5] — , "Gaussian interference channel capacity to within one bit," IEEE Trans. Inf. Theory, vol. 54, no. 12, pp. 5534-5562, Dec. 2008.

[6] İ. E. Telatar and D. N. C. Tse, "Bounds on the capacity region of a class of interference channels," in Proc. IEEE Int. Symposium on Information Theory (ISIT), Jun. 2007, pp. 2871-2874.

[7] X. Shang, G. Kramer, and B. Chen, "Outer bound and the noisyinterference sum-rate capacity for Gaussian interference channels," in Proc. 42nd Annual Conference on Information Sciences and Systems (CISS), Mar. 2008, pp. 385-389.

[8] A. Raja, V. M. Prabhakaran, and P. Viswanath, "The two user Gaussian compound interference channel," in Proc. IEEE Int. Symposium on Information Theory (ISIT), Jul. 2008, pp. 569-573.

[9] V. S. Annapureddy and V. V. Veeravalli, "Gaussian interference networks: Sum capacity in the low interference regime," in Proc. IEEE Int. Symposium on Information Theory (ISIT), Jul. 2008, pp. 255-259.

[10] E. Akuiyibo and O. Lévêque, "Diversity-multiplexing tradeoff for the slow fading interference channel," in Proc. IEEE Int. Zurich Seminar on Commun., Mar. 2008, pp. 140-143.

[11] C. Akçaba and H. Bölcskei, "Diversity-multiplexing tradeoff in fading interference channels," in preparation.

[12] B. Hassibi and B. Hochwald, "How much training is needed in multipleantenna wireless links?" IEEE Trans. Inf. Theory, vol. 49, no. 4, pp. 951963, Apr. 2003.

[13] L. Zheng and D. N. C. Tse, "Diversity and multiplexing: A fundamental tradeoff in multiple-antenna channels," IEEE Trans. Inf. Theory, vol. 49, no. 5, pp. 1073-1096, May 2003.

[14] T. Han and K. Kobayashi, "A new achievable rate region for the interference channel," IEEE Trans. Inf. Theory, vol. 27, no. 1, pp. 49-60, Jan. 1981.

[15] G. Kramer, "Review of rate regions for interference channels," in Proc. IEEE Int. Zurich Seminar on Commun., Feb. 2006, pp. 162-165.

[16] Y.-H. Nam and H. El Gamal, "Joint lattice decoding achieves the optimal diversity multiplexing tradeoff of multiple access channels," 2007 , submitted to IEEE Trans. Inf. Theory.

[17] M. Badr and J.-C. Belfiore, "Distributed space-time block codes for the non cooperative multiple access channel," in Proc. IEEE Int. Zurich Seminar on Commun., March 2008, pp. 132-135.

[18] P. Coronel, M. Gärtner, and H. Bölcskei, "Diversity-multiplexing tradeoff in selective-fading multiple-access MIMO channels," in Proc. IEEE Int. Symposium on Information Theory (ISIT), Jul. 2008, pp. 915-919. 